\title{
Cytogenetic characterisation of small round cell tumours using fine needle aspiration
}

\author{
C Sreekantaiah, L Appaji, D Hazarika
}

\begin{abstract}
Cytogenetic analysis of short term cultures of fine needle aspirates from two tumours, characterised cytologically as small round cell neoplasms, showed specific clonal chromosomal abnormalities. In both cases the cytogenetic finding of a $t(11 ; 22)(q 24 ; q 12)$ helped determine their diagnosis as peripheral neuroectodermal tumours of the thoraco-pulmonary region (Askin's tumour). These findings suggest that cytogenetics can reliably distinguish neoplasms which present as undifferentiated small round cell tumours.
\end{abstract}

Small round cell malignant tumours are histologically similar which makes their differential diagnosis difficult morphologically, especially, when the degree of differentiation of the tumour is poor. Precise diagnosis is important because different therapeutic approaches have been developed for the many forms of small round cell tumours. Ancillary diagnostic techniques such as special histological stains, electron microscopy, immunohistochemistry, immunofluorescence and tissue culture are then necessary to distinguish between the various neoplasms. Each of these techniques, however, has its own limitations. ${ }^{12}$

The chromosomal make-up of tumours provides yet another valuable diagnostic criterion which has not yet been extensively applied. Consistent and highly specific karyotypic abnormalities have been identified in several benign and malignant tumours in a situation akin to that of leukaemias. ${ }^{3}$ These cytogenetic findings could be diagnostically useful to pathologists when faced with a lack of specific evidence of differentiation in some tumours.

Fine needle aspiration biopsy is a simple, minimally traumatic, and accurate diagnostic procedure for the study of various neoplasms. It is widely used at present for the diagnostic workup of several tumours, including small round cell neoplasms, with excellent results. ${ }^{14}$ We report two cases in which cytogenetic analysis from such material aided in the differential diagnosis of small round cell neoplasms.

\section{Methods}

About $0.5 \mathrm{ml}$ of fine needle aspirates were collected aseptically from tumours in RPMI 1640 medium supplemented with $20 \%$ fetal calf serum and $2 \%$ antibiotics. The material was seeded into Falcon T-25 plastic culture flasks and cultured in the same medium at $37^{\circ} \mathrm{C}$ in $5 \%$ carbon dioxide. After 24 hours the unattached cells were harvested. The harvest procedure included exposure to colcemid $(2 \mu \mathrm{g} / \mathrm{ml})$ for one to two hours, hypotonic treatment (potassium chloride $3 \mathrm{~g} / \mathrm{l}$, EGTA $0.2 \mathrm{~g} / \mathrm{l}$, HEPES $4.8 \mathrm{~g} / \mathrm{l}$ ) for 30 minutes and overnight fixation in 3:1 (v/v) methanol:acetic acid. Flame-dried slides were GTG-banded and the karyotypes expressed according to the International System for Chromosome Nomenclature. ${ }^{3}$

Ultrasound guided fine needle aspiration biopsy (FNAB) was performed by standard techniques using a 22 gauge lumbar puncture needle fitted in a $10 \mathrm{cc}$ disposable plastic syringe and Cameco handle. ${ }^{5}$ Air dried and wet fixed (in 95\% alcohol), slides were prepared and stained with May-Grünwald-Giemsa and Papanicolaou methods, respectively.

After the unattached cells had been removed for cytogenetic harvest, fresh RPMI 1640 medium containing $20 \%$ fetal calf serum and $2 \%$ antibiotics was added to the flask and the cultures grown at $37^{\circ} \mathrm{C}$ in $5 \%$ carbon dioxide. The cultures were observed daily and the medium changed every three days. Good cell attachment and growth was observed in case 2.

\section{Case reports}

CASE 1

A 10 year old boy was investigated because of swelling in the left upper chest of one month's duration, proptosis of the left eye with frontotemporal swelling for 20 days, intermittent fever for a month and dyspnoea for 15 days. The swelling in the left anterior chest wall had progressively increased in size and at the time of presentation was $12 \times 10 \mathrm{~cm}$ large. A chest $x$-ray picture showed a homogeneous opacity in the left hemithorax with bony irregularity and periosteal reaction of the second, third, and fourth ribs. The skull showed a superficial lytic area in the left frontal bone. A bone scan showed diffusely spread disease in the skeletal system. Neuroblastoma with metastases to the left eye, or Ewing's sarcoma of the rib, was diagnosed.

Cytological examination of an FNAB sample showed darkly stained small round cells with pseudorosettes in a neurofibrillary background. The cytological diagnosis was consistent with peripheral neuroectodermal tumour (PNET).

Cytogenetic analysis of 24 hour cultures showed three clones of cells with the following 
Figure 1 G-banded representative karyotype from case 1 showing the $t(11 ; 22)$, trisomy 7 and 14 and tetrasomy of chromosome 8 (arrowheads).

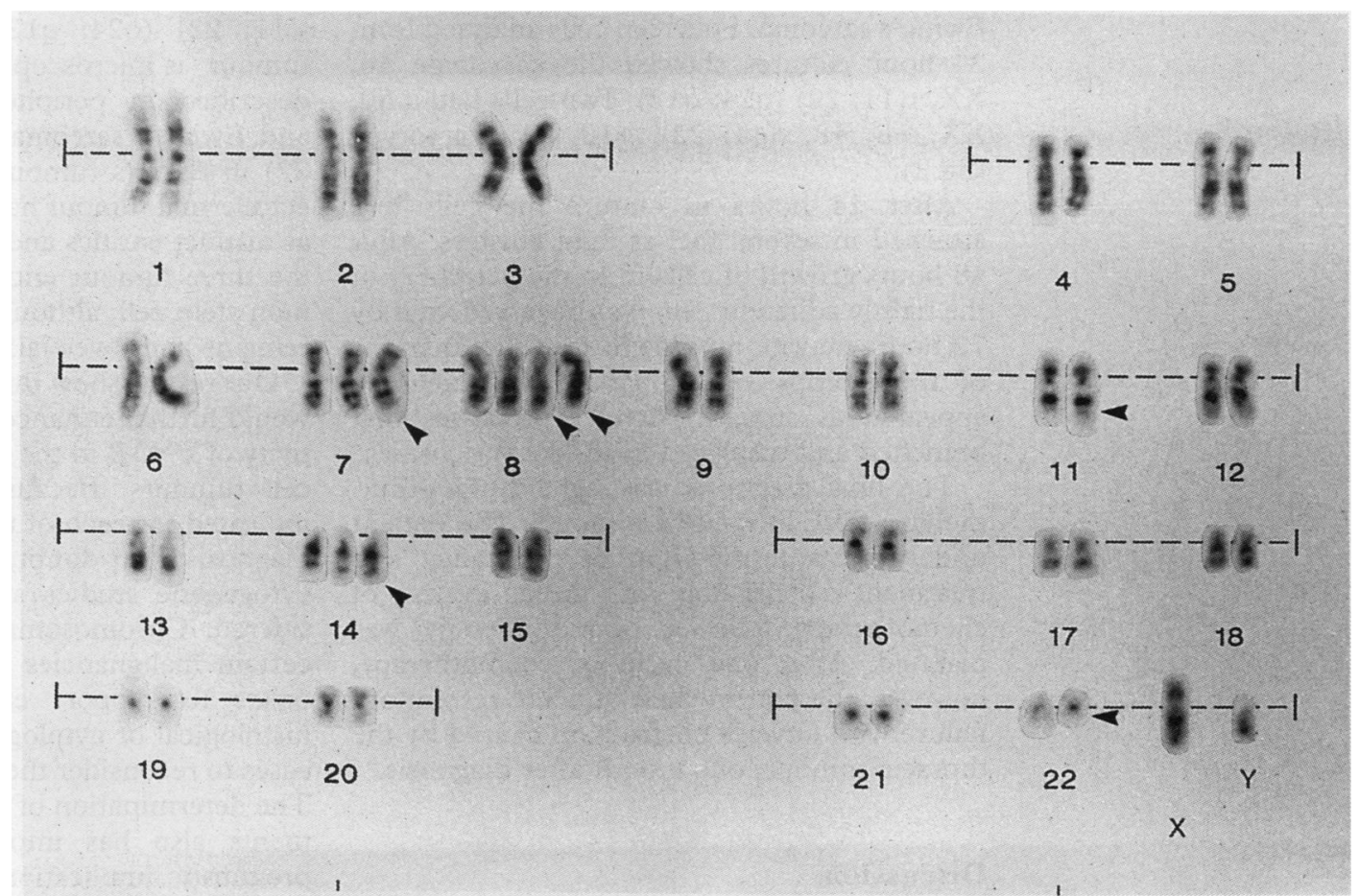

karyotypes; 5 cells, 46, XY, t(11; 22) (q24; q12)/5 cells, $47, X Y,+8, t(11 ; 22) / 3$ cells, 49 , $\mathrm{XY},+7,+8, \mathrm{t}(11 ; 22),+14 / 1$ cell, $50, \mathrm{XY},+7$, $+8,+8, t(11 ; 22),+14$ (fig 1$)$. The cytogenetic findings were suggestive of a diagnosis of Askin's tumour, PNET, or Ewing's sarcoma and not neuroblastoma, as the $t(11 ; 22)$ has been consistently noted in these tumour types. There was poor attachment and growth of the cells in tissue culture.

After clinical correlations a final diagnosis of PNET of the left thoraco-pulmonary region (Askin's tumour) with dissemination was made. The patient was treated with concurrent chemotherapy and radiotherapy to the chest and left orbit. He showed a good response.

CASE 2 A 20 year old woman presented with a history of chest pain, dyspnoea, and cough of two months' duration and a fever of eight days. At the time of presentation she was five months pregnant. A chest $x$-ray picture showed a mass on the right lung, $16 \times 14 \times 10 \mathrm{~cm}$ in size. An ultrasound scan of the chest showed a mass in the right lung with a necrotic area in the centre. Gross hepatomegaly and a single live fetus of 24 weeks' gestation with oligohydramnios was observed abdominally. A clinical diagnosis of mediastinal non-Hodgkin's lymphoma or extragonadal germ cell tumour of the mediastinum was considered.

An ultrasound guided FNAB of the mass showed a small cell neoplasm with neurofilaments and pseudorosettes. PNET of the thoraco-pulmonary region was diagnosed.

The cytogenetic results were consistent with a diagnosis of PNET, Askin's tumour, or,
Figure 2 G-banded representative karyotype from case 2 showing the $t(11 ; 22)$ abnormality (arrowheads).

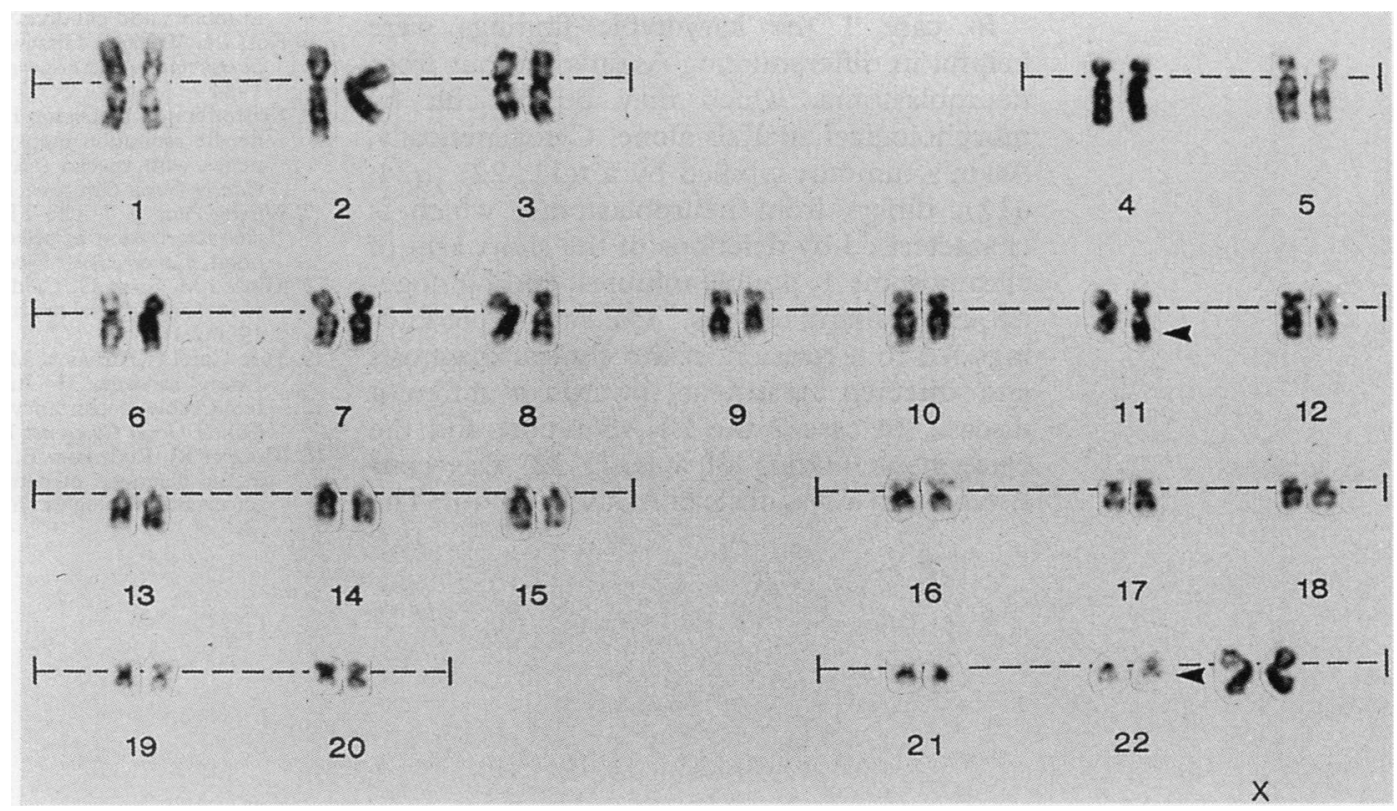


Ewing's sarcoma. Fourteen cells analysed from 24 hour cultures showed the karyotype 46, $\mathrm{XX}, \mathrm{t}(11 ; 22)$ (q24; q12). Two cells had a 50, $\mathrm{XX},+8,+8, \mathrm{t}(11 ; 22),+12,+19$ karyotype (fig 2).

After 24 hours in culture the cells had attached in several foci as tight clusters. After 48 hours growth of cells from the periphery of the tightly adherent cells was observed, and by 72 hours neurite outgrowth from the margins of the clumps was apparent. The neurites appeared as straight, slender processes that branched and displayed bead-like varicosities.

The final diagnosis was right thoraco-pulmonary PNET (Askin's tumour). The patient underwent a termination of pregnancy and treatment, consisting of three cycles of chemotherapy, followed by radiotherapy, was planned. After one cycle of chemotherapy, however, the patient died of acute respiratory failure with airways obstruction caused by the thoracic tumour, one month after diagnosis.

\section{Discussion}

Fine needle aspiration of tumour cells provides an excellent opportunity to procure material for chromosomal studies and is eminently suited to cancer cytogenetics. The aspirate contains viable cells representative of the tumour cell population and a comparatively small admixture of stromal and other supporting elements compared with an open surgical biopsy specimen or an excised tumour. The aspirate is received as a single cell suspension, obviating the necessity of mechanical and enzymatic disaggregation. The culture time is also considerably reduced, allowing faster cytogenetic diagnosis. If necessary, additional aspirates can also be obtained with little trauma to the patient. Despite the feasibility of chromosomal analysis from FNAB material the technique has not been widely applied in routine solid tumour cytogenetics. ${ }^{6}$ The two cases reported here indicate the success of the method as well as the usefulness of a combined cytological and cytogenetic approach in establishing a definitive diagnosis.

In case 1 the karyotypic findings were helpful in differentiating Askin's tumour from neuroblastoma, which may be difficult by morphological analysis alone. Cytogenetically, Askin's tumour, typified by a $t(11 ; 22)$ (q24; q12), differs from neuroblastoma, which is characterised by deletions of the short arm of chromosome 1, double minutes, and homogeneously staining regions. ${ }^{3}$ The karyotypic findings led to a revision of the clinical diagnosis and directed treatment towards a different disease. In case 2 the FNAB report and the cytogenetic finding of a $t(11 ; 22)$ was consistent with a diagnosis of Askin's tumour. The $\mathrm{t}(11 ; 22)$ (q24; q12) exhibited by Askin's tumour ${ }^{7}$ is microscopically identical with that described in peripheral neuroepithelioma ${ }^{78}$ and Ewing's sarcoma. ${ }^{79}$ The finding of $t(11$; 22) in Askin's tumour and peripheral neuroectodermal tumour has led to their recognition as distinct entities and also the suggestion that the three tumour entities derive from a common stem cell, although the exact histogenesis remains controversial.

Our results show that the use of cytogenetics would further enhance the accuracy and sensitivity of FNAB in the diagnosis of small round cell tumours. Because specific treatment is indicated for each of these tumours, when the diagnosis is in doubt, additional aspirates for cytogenetic studies should be strongly considered. Chromosomal markers diagnostic of certain malignancies can be used by pathologists to support either the diagnosis on histological or cytological grounds or in some cases to reconsider the histological diagnosis. ${ }^{10}$ The determination of chromosomal rearrangements also has important therapeutic and prognostic implications for the clinician with regard to the choice of and response to treatment, recurrence and metastatic potential of the tumour, as well as the survival of the patient. Cytogenetics is a powerful means of gaining insight into the biology of the disease as it forms a basis for most molecular studies which are crucially important to the understanding of the fundamental nature of the disease. The technique should therefore be used to expand the diagnostic armamentarium and improve the pathological interpretation of tumours.

We thank Mr Ameenulla Shariff and Mr Suresh Satpute fo technical assistance.

1 Akhtar M, Ali AM, Sabbah R, Bakry BSM, Nash JE. Fine needle aspiration biopsy diagnosis of round cell malignant tumors of childhood. Cancer 1985;55:1805-17.

2 Variend S. Small cell tumors in childhood: A review. $f$ Pathol 1985;145:1-25.

3 Sandberg AA. The Chromosomes in human cancer and leukemia. Second edition. New York: Elsevier Science Publishing Co, Inc., 1990.

4 Schaller RT, Schaller JF, Buschmann G, Kiviat N. The usefulness of percutaneous fine needle aspiration biopsy in infants and children. $\mathcal{F}$ Ped Surg 1983;18:398-405.

5 Koss LG, Woyke S, Olszewski W. Aspiration biopsy. Cytologic interpretation and histologic bases. New York: Igaku-Shoin 1984.

6 Kristoffersson U, Olsson H, Vendel E-M, Mitelman F. Fine needle aspiration biopsy: a useful tool in tumor cytogenetics with special reference to malignant lymphomas. Cancer Genet Cytogenet 1981;4:53-60.

7 Whang-Peng J, Triche TJ, Knutsen T, et al. Cytogenetic characterization of selected round cell tumors of childhood. Cancer Genet Cytogenet 1986;21:185-208.

8 Miozzo M, Sozzi G, Calderone C, et al. $t(11 ; 22)$ in three cases of peripheral neuroepithelioma. Genes Chrom Cancer cases of periphera

9 Turc-Carel C, Aurias A, Mugneret F, et al. Chromosomes in Ewing sarcoma. I. An evaluation of 85 cases and remarkable consistency of the $t(11 ; 22)$ (q12; q24) Cancer Genet Cytogenet 1988;32:229-38.

10 Motzser RJ, Rodriguez E, Reuter VE. Genetic analysis aid in the diagnosis of patients with midline carcinomas of uncertain histologies. $¥ N C I$ 1991;83:341-6. 